\title{
Chest Pain in the Emergency Room. Importance of a Systematic Approach
}

\author{
Roberto Bassan, Marcelo Scofano, Roberto Gamarski, Hans Fernando Dohmann, Lúcia Pimenta, \\ André Volschan, Mônica Araujo, Cristina Clare, Marcelo Fabrício, Carlos Henrique Sanmartin, \\ Kalil Mohallem, Sergio Gaspar, Renato Macaciel
}

Rio de Janeiro, RJ

\begin{abstract}
Objective - To evaluate the efficiency of a systematic diagnostic approach in patients with chest pain in the emergency room in relation to the diagnosis of acute coronary syndrome (ACS) and the rate of hospitalization in high-cost units.

Methods - One thousand and three consecutive patients with chest pain were screened according to a preestablished process of diagnostic investigation based on the pre-test probability of ACS determinate by chest pain type and ECG changes.
\end{abstract}

Results - Of the 1003 patients, 224 were immediately discharged home because of no suspicion of ACS (route 5) and 119 were immediately transferred to the coronary care united because of ST elevation or left bundle-branch block ( $L B B B)$ (route 1) (74\% of these had a final diagnosis of acute myocardial infarction [AMI]). Of the 660 patients that remained in the emergency room under observation, 77 (12\%) had AMI without ST segment elevation and 202 (31\%) had unstable angina (UA). In route 2 (high probability of ACS) $17 \%$ of patients had AMI and $43 \%$ had UA, whereas in route 3 (low probability) $2 \%$ had AMI and $7 \%$ had UA. The admission ECG has been confirmed as a poor sensitivity test for the diagnosis of AMI (49\%), with a positive predictive value considered only satisfactory (79\%).

Conclusion - A systematic diagnostic strategy, as used in this study, is essential in managing patients with chest pain in the emergency room in order to obtain high diagnostic accuracy, lower cost, and optimization of the use of coronary care unit beds.

Key-words: acute myocardial infarction, chest pain, emergency room, unstable angina

Hospital Pró-Cardíaco e PROCEP - Centro de Ensino e Pesquisas do Pró-Cardíaco - Rio de Janeiro

Mailing address: Roberto Bassan - Hospital Pró-Cardíaco - Rua Dona Mariana, 219 - 22280-020 - Rio de Janeiro, RJ
"Thus, in this era of economic constraints, we may find to preserve the coronary care unit, we just know when to use it". Lee TH \& Goldman L1.

Chest pain is certainly one of the most common reasons for needing medical assistance in the emergency rooms. It is estimated that in the United States about 3-6 million emergency room admissions due to chest pain are carried out, which corresponds to about 5-10\% of the total admissions ${ }^{2-4}$. In Brazil, we do not have information or estimates, but our institution only, registered 515 emergency admissions due to chest pain during the period of November 1966 to June 1997, which is 27,7\% of the total 2490 consultations during that period, the most frequent reason for going to the emergency room.

Although a number of diseases cause chest pain, those originating in the cardiovascular system are the ones that worry physicians as well as patients the most. This is explained by the greater mortality risk and the need for hospitalization. However, only $10-15 \%$ of the patients who go to the emergency room with chest pain have acute myocardial infarction, and less than $1 \%$ have pulmonary embolia or aortic dissection ${ }^{4,5}$, but more than $50 \%$ are hospitalized for diagnostic investigation (to exclude acute myocardial infarction) ${ }^{1,4,6,7}$. The cost of hospitalization varies in the United States from U $\$ 2000$ to U $\$ 5000$ per patient ${ }^{8,9}$, thus, equaling a total annual cost of approximately U\$6-8 billions just to exclude the diagnosis of acute myocardial infarction ${ }^{10}$. In addition, it is estimated that $5 \%$ of patients with chest pain and acute myocardial infarction are erroneously sent home without a diagnosis being made in the emergency room ${ }^{4,11,12}$, which has resulted in great expenses for physicians and hospitals in the US in malpractice law suits, (in Brazil, although no statistical data are available, one can assume this rate to be higher, due to the simple fact that in many emergency rooms no electrocardiograph or cardiologist, and often neither are present). The mortality, within a short period, of these patients, inappropriately sent home is 
significantly higher than mortality in those initially hospitalized, in spite of being younger and having a lower rate of angina or previous infarction ${ }^{11}$.

All these facts point to the necessity of establishing accurate methods and criteria for rapid identification of high risk patients to treat them precociously and appropriately, and those with no heart disease, to immediately release them, safely and at low cost.

\section{Methods}

The Hospital Pró-Cardíaco is a private institution for primary and tertiary assistance in internal medicine and cardiology, located in down-town Rio de Janeiro. It has an active 9 bed emergency room and a home care system that uses ambulances. Both are staffed by cardiologists. The majority of emergency consultations are for clinical diseases usually of cardiovascular, neurological or respiratory etiology and not for surgical issues. The emergency room is backed-up by a quick clinical analysis laboratory, capable of supplying CK-MB levels myoglobin and troponina assays (as well as other tests) in less than 2 hours in addition to a 24 hours, round-the-clock echocardiography service.

With the objective of improving the quality of assistance to patients with complaints of chest pain and to standardize the diagnosis and the therapy, a systematic diagnostic protocol was created for these patients, according to their probability of having acute coronary syndromes. The systematic diagnostic investigation model was obtained through a consensus of a group of researchers after extensive revision and discussion of same models already proposed in the medical literature ${ }^{5,6,10,13}$. When creating this model, not only the functional characteristics of our institution were taken into consideration, but also the necessity for a diagnosis as accurate as possible, and time cost efficiency. Patients were selected by analyzing the type of chest pain and the characteristics of the admission electrocardiogram. With these data patients were enrolled in a diagnostic path - denominated routes - in which the necessity and the duration of investigation of a possible coronary etiology was made according to the estimated probability of the disease.

Chest pain was considered when it occurred anywhere between the nose and the umbilical button and constituted a spontaneous complaint from the patient. To be included in the protocol, the patient had to esperience severe chest pain anytime from 12 hours to admission to the hospital.

The cardiologist in the emergency room prospectively and carefully asked the patient to describe all characteristics of the chest pain and litled a proper form. From there on - and before the first electrocardiogram - the pain was classified by the attending physician as one of the following four types: 1) type A (a definite angina pain): thoracic pain with characteristics that gave the physician a certainty of an acute coronary syndrome diagnosis, independent of the results of complementary exams; 2 ) type $B$ (probably angina pain): chest pain with characteristics that made acute coronary syndrome the main diagnostical hypothesis, however requiring complementary exams for confirmation of the diagnosis; 3 ) type $\mathrm{C}$ (probably not angina pain): chest pain with characteristics that did not make acute coronary syndrome the main diagnostic hypothesis, however, requiring complementary exams to exclude the diagnosis; 4) type $\mathrm{D}$ (definitely not angina pain): chest pain with characteristics that did not include acute coronary syndrome in the differential diagnosis $(-\mathrm{D} 1=$ with etiologic diagnosis not determined at admission. D2 = with determined etiologic diagnosis).

After classification of the type of chest pain, an 18 lead electrocardiogram was made ( 12 conventional plus 4 right precordial and 2 dorsal) - the electrocardiograms were classified as four types: 1) electrocardiogram with ST elevation; in the case of positive deviation of J-ST $>0.1 \mathrm{mV}$ in a least 2 leads of the frontal plan, or $>0.2 \mathrm{mV}$ in at least two leads of the horizontal plan; 2) electrocardiogram with ST depression or inversion of $\mathrm{T}$ : in case of negative deviation of J-ST $\geq 0.1 \mathrm{mv}$ in at least two leads, or an isolated inversion of $\mathrm{T}$ in at least two leads; 3 ) electrocardiogram with left bundle-branch block: when in sinusal rhythm, there would be QRS complexes with duration $\geq 120 \mathrm{~ms}$, with QS or RS morphology in $\mathrm{V} 1$ and intrinsic deflection $\geq 60 \mathrm{~ms}$ in I, V5 or V6, associated with the absence of Q waves in leads ${ }^{14}, 4$ ) Normal or nonspecific electrocardiogram: no alteration occurred, or when the alterations in duration or QRS morphology and/or the alterations of the J-ST and T position were of lesser intensity than described above, even in the presence of previous pathologic $Q$ waves.

In patients who had left bundle-branch block in the electrocardiogram, the chest pain was classified afterwards as 2 types: 1) the pain of acute myocardial infarction: chest pain that suggests acute myocardial infarction by its clinical characteristics, principally by its strong intensity and prolonged duration. ( $>30 \mathrm{~min}$ ), and by other symptoms (paleness, transpiration, nausea, vomiting, shortness of breath, etc);2) the pain of no acute myocardial infarction: when the chest pain does not have the above characteristics.

Based on the type of pain information and the type of admission electrocardiogram, the patients were enrolled to diagnostic strategies or routes, in which determination of diagnostic procedures and prognosis and therapeutic measures were pre-determined (fig.1).

As such, we initially enrolled patients into route 1 who had chest pain and an electrocardiogram strongly suggesting acute myocardial infarction (suggestive pain + electrocardiogram with ST elevation or left bundle-branch block). Due to the very high probability of acute myocardial infarction, these patients underwent either to trombolysis, primary angioplasty or just the conservative emergency room treatment and, soon after, were transferred to the coronary unit; we sent into route 2 those with electrocardiographic changes of ST depression or inversion of T, or those with chest pain suggestive of acute coronary syndrome (type A or B) but without electrocardiographic changes of myocardial ischemia. Due to the high probability of unstable angina 


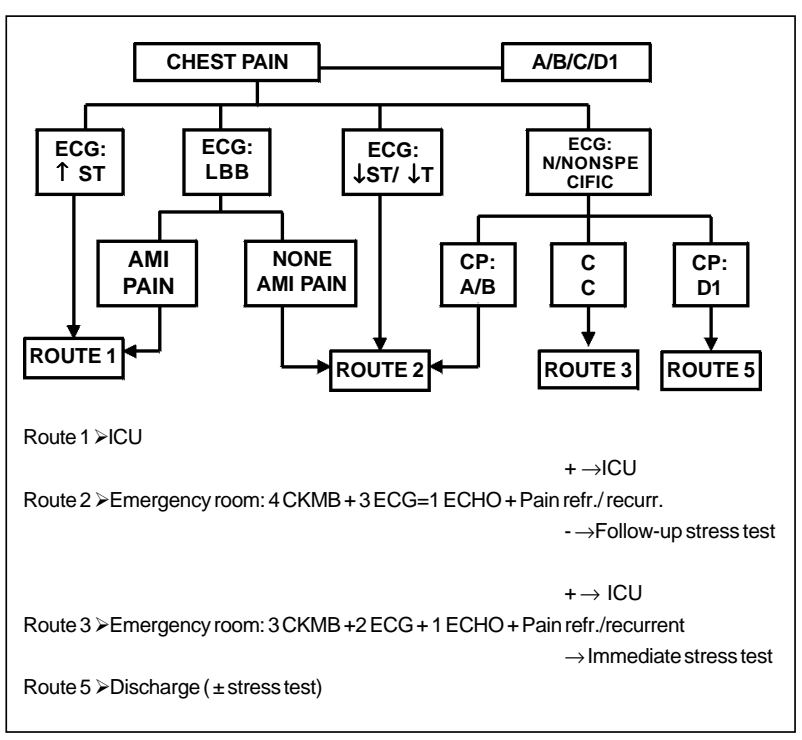

Fig.1 - Flowchart of attendance of patients with chest pain. LBBB- left bundlebranch block; CKMB- creatine kinase - MB; CP- chest pain; ECG- electrocardiogram; ERGO- ergometric test; AMI- acute myocardial infarction; N/AMI = INESP- normal nonspecific; refractory/current; SE- emergency room; UCcoronary unit.

and the moderate probability of acute myocardial infarction, these patients remained in the emergency room, had to electrocardiographic and serial enzyme tests $(3 / 3 \mathrm{~h})$ in the following 9 hours, and an echocardiogram; we enrolled patients into route 3 those with chest pain not completely excluding acute coronary syndrome (type C) and with absence of electrocardiographic changes due to myocardial ischemia. Due to the low probability of unstable angina and acute myocardial infarction, these patients also remained in the emergency room and underwent electrocardiographic evaluations and serial enzyme tests $(3 / 3 \mathrm{~h})$ in the following 6 hours and an echocardiogram during this period.

Those patients enrolled to routes 2 and 3 who during the evaluation period in the emergency room had any evidence of myocardial necrosis (enzymatic elevation) or persistent or recurring myocardial ischemia, were transferred to the coronary unit. The remaining patients in route 2 continued under surveillance in the emergency room or in the intermediate care ward, and if they remained symptomatic but without evidence of recurrent ischemia, they underwent to a stress test (or myocardial perfusion scintilography) within a $24-48$ hours period. The patients initially enrolled to route 3 and who also did not have necrosis or myocardial ischemia during the evaluation period in the emergency room also underwent to the exercise test within 9-12 hours after admission.

Route 4 was reserved for evaluation of patients with suspicion of thoracic vascular disease (pulmonary embolus, aortic dissection), this way patients underwent specific examinations, mainly imaging, therefore were not be included in this study.

Patients were enrolled into route 5 had non-anginal chest pain (type D) and with no electrocardiographic changes of myocardial ischemia. These patients were released immediately from the emergency room with the recommendation that they undergo a follow-up exercise test.

For transfer of a patient initially enrolled to route 2 and 3 to the coronary unit, the patient had to have an of serum CK-MB above the highest limit considered normal in any one of the serial tests carried out in the respective route even if the elevation did not meet the diagnostic criteria for acute myocardial infarction, which was considered myocardial necrosis. Transfer to the coronary unit also happened with the worsening in ST depression or the inversion of T, the appearance of ST-T alterations not pre-existent in serial electrocardiograms, made in the respective route, or the demonstration, of partial or total in contractile changes in the bidimensional echocardiogram in areas that did not show Q waves in the electrocardiogram, all considered signs of persistent or recurrent myocardial ischemia. The resistance of chest pain to the proper anti-ischemic medication, or its recurrence, although without electrocardiographic changes were also considered criteria for transfer of patients from routes 2 and 3 to the coronary unit.

With regard to the final diagnosis, the patient with acute myocardial infarction was determined to be the one who had, apart from the characteristics of the admission electrocardiogram, abnormal CK-MB $>10 \%$ of total CK and accompanied by at least one of the following criteria: 1) three consecutive measurements of CK-MB above the upper limit of normalcy; 2) two consecutive measurements of CK-MB with values at least $50 \%$ above the upper limit of normality; 3) a measurement of CK-MB with value at least $100 \%$ over the upper limit of normality.

Patients with unstable angina were those who did not have any characteristics of CK-MB elevations mentioned present but did have: 1) type A or B chest pain, with duration $>20 \mathrm{~min}$., independently of changes in the electrocardiogram, the echocardiogram or in the exercise test; or 2) type $\mathrm{C}$ or $\mathrm{D}$ chest pain, duration $>20 \mathrm{~min}$., but also wiht ischemic alterations in the electrocardiogram, in the echocardiogram or in the exercise test.

Patients who did not fulfill any of the diagnostic criteria of acute myocardial infarction or unstable angina were designated as non-bearers of acute coronary syndrome.

Finally, patients in whom the diagnosis of acute myocardial infarction, unstable angina or absence of acute coronary syndrome was neither ruled of nor confirmed due to insufficient diagnostic information, were designated as non bearers of acute coronary syndrome.

For accuracy calculation of diagnostic tests (chest pain, electrocardiogram and echocardiogram), patients with an indeterminate final diagnosis were excluded, so as not to introduce biases into the results. Values supplied as mean are followed by the corresponding standard deviation. The significance of difference of proportions was calculated by the qui-square test.

\section{Results}

From November/1996 to February/1998, 1003 consecutive patients with the principal complaint of chest pain 
were treated in the emergency room and they constitute a sample of this prospective study. Ages varied between 1490 years $(60.3 \pm 15,5), 632(63 \%$ were men and $258(26 \%)$ hada previous history of coronary disease.

Chest pain, type A (definitely angina) was observed in $225(22 \%)$ patients, type B (probably angina) in $287(29 \%)$, type $\mathrm{C}$ (probably not angina) in $268(27 \%)$ and type D (definitely not angina) in 223 (22\%).

The admittance electrocardiogram (available in 971 patients) showed ST elevation in 106 (11\%), ST depression or inversion of T wave in $159(16 \%)$, left bundle-branch block in 38 (4\%) and absence of these changes (normal or nonspecificECG) in 668(69\%).

Of 1003 patients studied, 165 (16\%) had the final diagnosis of acute myocardial infarction, 225 (22\%) of unstable angina, 324 (32\%) of the absence of acute coronary syndrome and $289(29 \%)$ had an indefinite diagnosis. The demographic and clinical characteristics of these patients are on table I. It should be noted that patients with a diagnosis of acute myocardial infarction and unstable angina had a greater prevalence of risk factors for coronary disease than the others.

Of 1003 patients, 224 (22\%) were immediately discharged as the chest pain was considered definitely not angina and the electrocardiogram normal or nonspecific (route 5). 119 patients, (12\%) were immediately transferred to a coronary unit because they had ST elevation or left bundlebranch block (route 1). The other 660 patients remained in the emergency room and were enrolled to routes 2 or 3 , according to the admission electrocardiogram and the type of chest pain, for diagnostic investigation and risk stratification. Of these, 222 (34\%) later developed some criteria for transferral to the coronary unit. They either showed some elevation in myocardial necrosis markers or had important new or recurrent myocardial ischemia in the electrocardiogram or in the echocardiogram (many had two or more criterias). Therefore, from the total of 336 patients with criteria for hospitalization in the coronary unit by the flowchart utilized (routes 1, 2 and 3), 165 left with a final diagnosis of acute myocardial infarction and 160 with the diagnosis of unstable angina ( 11 had an indeterminate diagnosis).

Of 119 patients who were initially in route 1 and moved to a coronary unit, 106 had ST elevation in the admission electrocardiogram and 13 with left bundle-branch block with chest pain suggestive of acute myocardial infarction. Of these, $88(74 \%)$ had the final diagnosis of acute myocardial infarction and $20(17 \%)$ of unstable angina. Of 433 patients who were selected to the emergency room for 9 hours, 73 (17\%) had the final diagnosis of acute myocardial infarction and $186(43 \%)$ of unstable angina. Of the 227 patients in to route 3 and investigated in the emergency room for 3 hours, $4(2 \%)$ had the final diagnosis of acute myocardial infarction and $16(7 \%)$ of unstable angina ( fig. 2 ).

The importance of the admission electrocardiogram in the final diagnosis of patients with chest pain can be evaluated in this study. Likewise, of 106 patients with ST elevation in the first electrocardiogram, $81(76 \%)$ had acute

\begin{tabular}{|c|c|c|c|c|}
\hline \multicolumn{5}{|c|}{$\begin{array}{l}\text { Table I - Demographic and clinical characteristics of } 1003 \text { patients } \\
\text { with chest pain according-to the final diagnosis }\end{array}$} \\
\hline & $\begin{array}{c}\text { AMI } \\
(\mathrm{n}=165)\end{array}$ & $\begin{array}{c}\text { UA } \\
(\mathrm{n}=225)\end{array}$ & $\begin{array}{l}\text { Non-ACS } \\
(\mathrm{n}=324)\end{array}$ & $\begin{array}{r}\text { Indet } \\
(\mathrm{n}=289)\end{array}$ \\
\hline Age & $66 \pm 13$ & $67 \pm 13$ & $51 \pm 14$ & $62 \pm 15$ \\
\hline Men & $76 \%$ & $59 \%$ & $64 \%$ & $58 \%$ \\
\hline Men $>60$ years & $50 \%$ & $34 \%$ & $15 \%$ & $29 \%$ \\
\hline Women $>70$ years & $16 \%$ & $26 \%$ & $6 \%$ & $17 \%$ \\
\hline CAD history & $35 \%$ & $46 \%$ & $8 \%$ & $24 \%$ \\
\hline Diabetes history & $16 \%$ & $20 \%$ & $4 \%$ & $8 \%$ \\
\hline Chest pain A/B & $92 \%$ & $90 \%$ & $20 \%$ & $32 \%$ \\
\hline ECG normal/non specific & $18 \%$ & $56 \%$ & $91 \%$ & $85 \%$ \\
\hline \multicolumn{5}{|c|}{$\begin{array}{l}\text { UA- unstable angina; CAD- coronary artery disease; ACS- acute coronary } \\
\text { syndrome; Indet- indeterminate; ECG- electrocardiogram; AMI- acute } \\
\text { myocardial infarction. }\end{array}$} \\
\hline
\end{tabular}

myocardial infarction, 15 (14\%) unstable angina, $6(6 \%)$ absence of acute coronary syndrome and 4 (4\%) an indeterminate diagnosis. On the other hand, from 165 patients with acute myocardial infarction, $81(49 \%)$ had ST elevation in the first electrocardiogram, $45(27 \%)$ ST depression or inversion of T, $9(5 \%)$ left bundle-branch block and $30(18 \%)$ normal or nonspecific electrocardiogram. Likewise, the sensitivity of ST elevation in the admission electrocardiogram for infarction was of $49 \%$, with a specificity of $96 \%$, a positive predictive value of $79 \%$ and a negative predictive value of $86 \%$.

Tables II and III illustrate the relation between the type of chest pain and the types of admission electrocardiogram and the final diagnosis. As was expected, the A/B type of chest pain was strongly related to the diagnosis of acute myocardial infarction and unstable angina, significantly different from pain types C/D. In regards to the electrocardiogram, a great majority of the patients with ST elevation had acute coronary syndrome (76\% of them had acute myocardial infarction). Of the patients who showed depression of ST/inversion of T, or both, a majority also had acute coronary syndrome $(28 \%$ had, as a matter of fact, acute myocardial infarction). Likewise, almost two thirds of patients with left bundle-branch block also had acute coronary syndrome (24\% of the total had enzymatic criteria of acute myocardial infarction). Finally, almost one fourth of the patients who initially had normal or non specific electrocardiograms, had a final of a diagnosis of acute coronary syndrome (4\% had acute myocardial infarction).

Bidimensional echocardiogram was routinely used in admission of patients enrolled to routes 1, 2 and 3 as a complementary diagnostic method of acute coronary syndrome. Alterations of contractility (partial/segmental) or global without pathological Q wave in electrocardiogram were found in $206(36 \%)$ of 576 patients who had echocardiograms and $168(82 \%)$ had the diagnosis of acute coronary syndrome ( $51 \%$ of these had acute myocardial infarction). On the other hand, of 370 patients with normal echocardiograms 148 (40\%) had acute coronary syndrome $(11 \%$ had acute myocardial infarction $)(\mathrm{p}<0.0001)$.

Figures 3 and 4 demonstrate chest pain accuracy from 


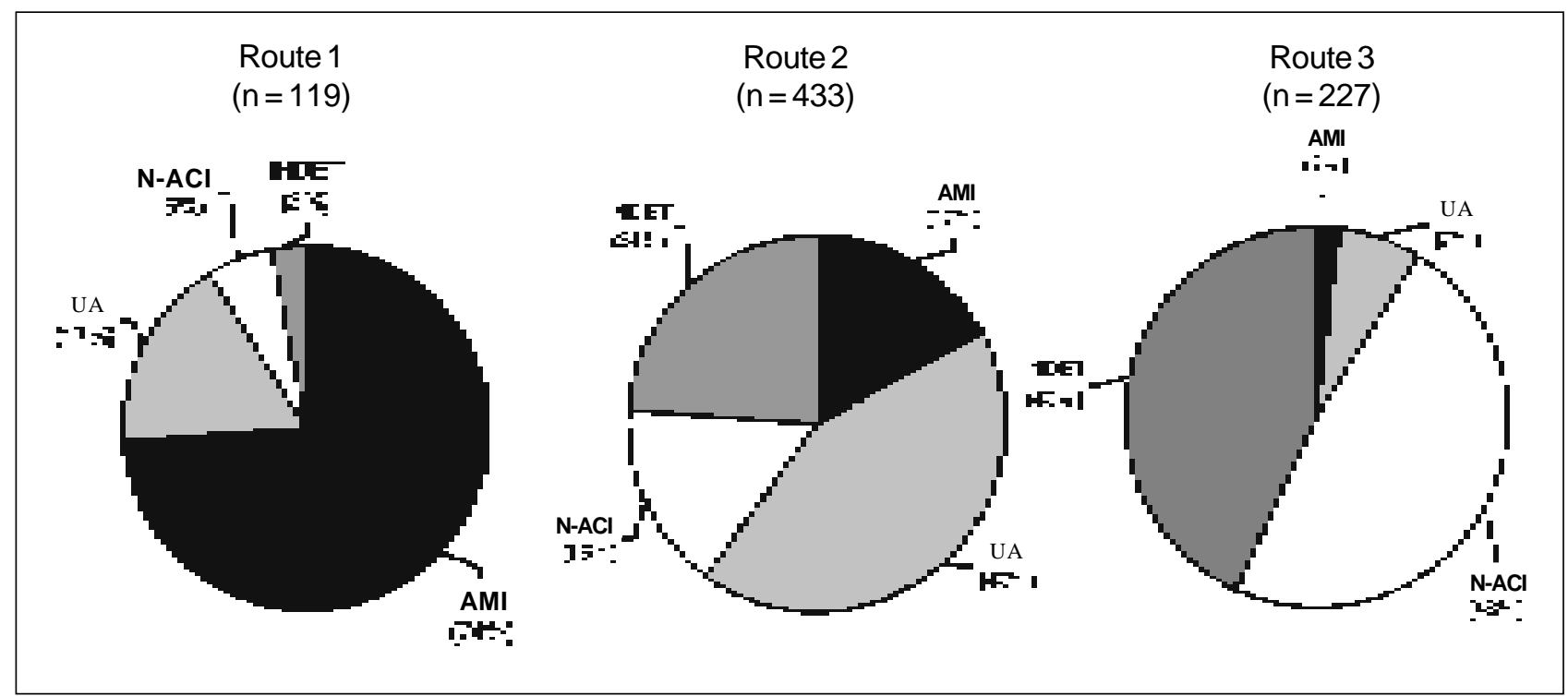

Fig. 2 - Distribution of acute myocardial infarction (AMI), unstable angina (UA), indeterminate diagnostic (Indet) and absences of acute coronary syndrome (N-ACS) in routes followed by 1003 patients with chest pain.

first electrocardiogram and first echocardiogram to the diagnosis of acute myocardial infarction in the emergency room (patients with non-determined diagnosis, were excluded from this analysis). As can be seen, A/B type chest pain had excellent sensitivity and negative predictive value (indicating very few false negative), but poor specificity and positive predictive value (indicating many false-positive cases), which is explained by the fact that the majority of patients with false-positive readings for infarction actually had unstable angina. The electrocardiogram had poor sensitivity (many false-negatives) whereas the echocardiogram had regular ratings in these indices.

\section{Discussion}

The policy of admitting the majority of patients to the coronary unit who are treated in the emergency room for chest pain, even if they do not have electrocardiographic changes, has been the practice during the last decades, due to the concern about erroneously discharging a patient who has acute coronary syndrome. Because the majority of these patients (60-80\%) not have acute myocardial infarction or unstable angina ${ }^{1,4,5,6,15}$ and the cost to eliminate these diagnoses is very high, a change in this policy has

\begin{tabular}{|c|c|c|c|c|}
\hline \multicolumn{5}{|c|}{$\begin{array}{l}\text { Table II - Relation between type of chest pain and final diagnosis } \\
\text { in } 1003 \text { patients examined in the emergency room }\end{array}$} \\
\hline & AMI & Non-ACS & Indet & $\mathrm{p}$ \\
\hline Pain type $A / B(n=512)$ & $69 \%$ & $13 \%$ & $18 \%$ & \multirow{2}{*}{$<0.0000$} \\
\hline Pain type $C / D(n=491)$ & $07 \%$ & $53 \%$ & $40 \%$ & \\
\hline $\begin{array}{l}\text { AMI- acute miocardial ir } \\
\text { acute coronary syndrome }\end{array}$ & $\begin{array}{l}\text { rction; } \\
\text { det- in }\end{array}$ & $\begin{array}{l}\text { - coronary } \\
\text { minate; } \mathrm{EC}\end{array}$ & $\begin{array}{l}\text { tery di } \\
\text { electro }\end{array}$ & $\begin{array}{l}\text { ise; ACS- } \\
\text { ardiogram }\end{array}$ \\
\hline
\end{tabular}

been recommended by various authors and several medical institutions in the last few years ${ }^{1,16-18}$.

Therefore, during the 1980s, the so-called Chest Pain Units were created, with the greater objective of increasing the diagnostic accuracy in patients with chest pain in the emergency room and to speed up the diagnostic and therapeutic approaches in these patients ${ }^{19-21}$. In the same way, algorithms or clinical decision trees and probability evaluations of the disease have been created by several authors to help in this task, besides identifying those who need to or need not to be transferred to a coronary unit or to undergo expensive examinations ${ }^{5,6,17,22-25}$. Consequently, this has resulted in the evaluation and treatment of many coronary patients changed from being in the coronary units to intermediate units or to the emergency rooms.

The final result of this policy of careful investigation of these patients in the emergency room has been very favorable. A significant reduction in the number of cases of acute myocardial infarction, which would not have been diagnosed by the conventional investigation model, has been one of the most important results obtained ${ }^{4,10,12,26}$. In addition to this, a smaller rate of hospitalization in the coronary unit of patients non-bearers of acute myocardial infarction or unstable angi-

\begin{tabular}{|c|c|c|c|c|}
\hline \multicolumn{5}{|c|}{$\begin{array}{l}\text { Table III - Relation between admission electrocardiogram and final } \\
\text { diagnosis in } 1003 \text { patients with chest pain examined in the emergency room }\end{array}$} \\
\hline & AMI & Non-ACS & Indet & $\mathrm{p}$ \\
\hline ST elevation $(n=106)$ & $90 \%$ & $6 \%$ & $4 \%$ & \\
\hline ST-T depression $(n=159)$ & $71 \%$ & $12 \%$ & $17 \%$ & $<0.0000$ \\
\hline $\operatorname{LBBB}(\mathrm{n}=38)$ & $63 \%$ & $11 \%$ & $26 \%$ & \\
\hline Normal/nonespecific $(n=668)$ & $23 \%$ & $42 \%$ & $35 \%$ & \\
\hline
\end{tabular}




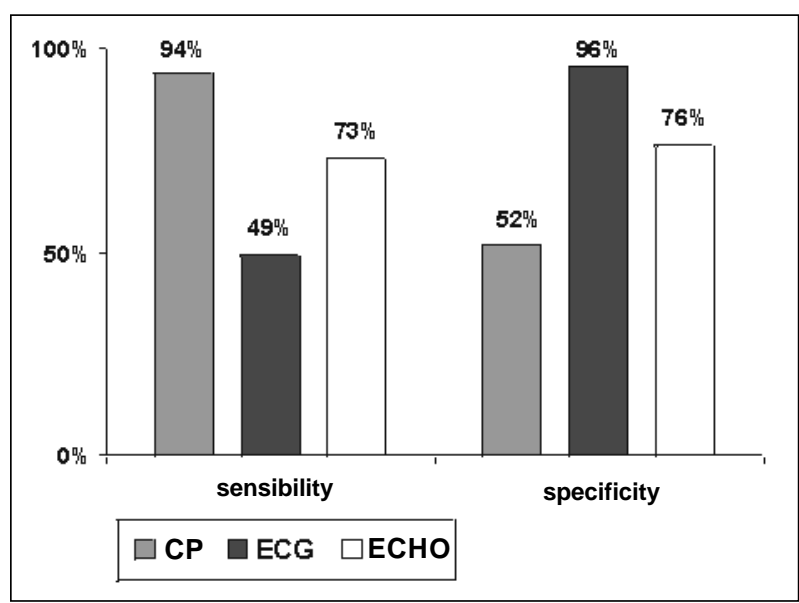

Fig. 3 - Sensiblility and specificity of type of chest pain (CP), of first electrocardiogram (ECG) and of first echocardiogram (ECHO), (see definition of positive in Methods), for diagnostic of acute myocardial infarction in $714 \mathrm{pa}$ tients with chest pain in emmergency room (excluded patients with final indeterminate diagnosis.

na has also been observed ${ }^{5,22,27,28}$ as well as a reduction in the length of hospitalization ${ }^{8,28,29}$. These facts have been shown to be responsible for the considerable reduction in hospital costs, of 40-60\% without an increase in the rate of cardiovascular events inside or outside of the hospital ${ }^{8,9,28,29}$.

Some authors have demonstrated that it is possible to identify subgroups of patients with chest pain and low risk of complications, using a clinical history and the electrocardiogram ${ }^{5,25,30}$. These patients may be admitted to lowcost units without incurring an increase risk, which improves the cost-benefit ratio. In fact, the coronary units have not been considered as cost-efficient for patients with chest pain, when the probability of acute myocardial infarction is less than $5-10 \%{ }^{15}$.

The Chest Pain Project of the Hospital Pró-Cardíaco created a systematized attendance model (flowchart) based on the evaluation of two parameters, simple to obtain in the emergency room: 1) the type of chest pain and 2) the alterations of the electrocardiogram. These parameters have already been validated in the medical literature as forewarnings of acute coronary syndrome, and reason to triage patients to separate investigation routes (fig.1).

The greater objective of establishing the routes is to permit reaching the diagnosis of acute myocardial infarction, unstable angina or absence of the acute coronary syndrome with the greater possible degree of certainty (probability of disease) and to establish the chances of events or cardiac complications, immediate or future (risk of events). In addition, this strategy also aims to accelerate the obtainment of a diagnosis, reduction in time spent in the emergency room or hospital for those with no acute coronary syndrome and only forward to the coronary unit the patients with acute myocardial infarction and unstable angina (who are the ones that can best benefit from this high cost unit).

Likewise, route 1 included patients with high initial probability of acute myocardial infarction due to the ST elevation or left bundle-branch block (associated with chest pain suggestive of infarction). This strategy resulted in more than $90 \%$ of patients initially hospitalized in coronary units effectively having a final diagnosis of acute coronary syndrome.

Patients with a high probability of acute coronary syndrome, however without the typical electrocardiographic changes of acute myocardial infarction, were enrolled to route 2 ; while remaining in the emergency room, under strict observation, their diagnoses were made in the following few hours. Of 433 patients on to route $2,73(17 \%)$ had acute myocardial infarction without elevation of ST and $186(43 \%)$ had unstable angina. So, more than half of the patients in route 2 had acute myocardial infarction, but only a part of these ( the ones with infarction and the ones with high or medium risk of unstable angina) were transferred to a coronary unit. This strategy once again favored the policy of only using the high cost unit for the patients with high risk acute coronary syndrome.

Route 3 included patients with low probability of acute coronary syndrome classified by the type of pain and no specific changes in the initial electrocardiogram.

Some patients with transient acute coronary syndrome or of little significance (low probability and low risk) and many patients with no coronary disease were included. Of 227 patients on route 3 , only $4(2 \%)$ had acute myocardial infarction (forwarded to coronary unit) and $16(7 \%)$ had unstable angina. These data indicates that route 3 received a smaller number of patients with acute coronary syndrome, but even so, a surveillance was necessary so as not to overlook these few cases, that eventually could be discharged home due to atypical symptoms and non-specific changes in the electrocardiogram.

Chest pain is a classic symptom of coronary disease and of myocardial ischemia. However, several cardiac or noncardiac diseases may cause chest pain, which makes the differential diagnosis of these diseases a great challenge. Diamond and Forrester ${ }^{31}$ demonstrated that the type of chest pain is the best determination indicative of pre-test probability of coronary disease in these patients, more

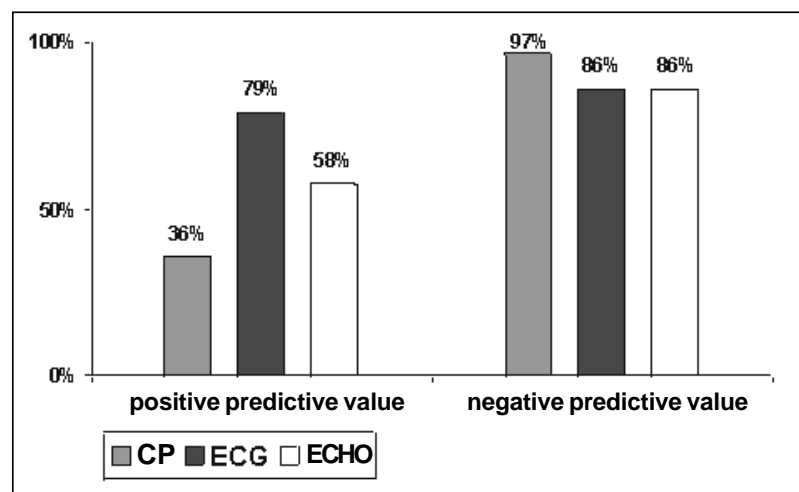

Fig. 4 - Predictive, positive and negative values of the chest pain type (CP) and of first electrocardiogram and of first echocardiogram (ECHO) (see definition of positive in Methods for acute myocardial infarction diagnosis) in 714 patients with chest pain in the emmergency room (excluded the patients with final indeterminate diagnosis). 
predictive than the basic or stress electrocardiogram. However, the diagnosis of unstable angina is frequently troublesome, as the electrocardiogram many times is normal in these patients. The US National Institutes of Health Guideline for treating unstable angina recognizes that the identification of the clinical manifestation of coronary disease is complex, and uses the characteristics of chest pain (typical or not) and duration of pain (more or less than $20 \mathrm{~min}$ ) to establish the diagnosis ${ }^{32}$.

Goldman et $\mathrm{al}^{5}$ and Lee et $\mathrm{al}^{23}$ proposed an algorithm for evaluation of patients with chest pain, where the first parameter to be checked is the presence or absence of ST elevation in the admission electrocardiogram. When this is present, the probability of acute myocardial infarction is $77 \%$ (in our study it was $76 \%$ ) and when absent, the characteristics of pain have major importance in the determination of probability of infarction. In addition, these authors divided the patients without ST elevation into high-risk $(>7 \%)$ and low-risk $(<7 \%)$ groups for acute myocardial infarction and recommended that the first lot be investigated in the coronary unit and the others in a unit of lesser surveillance and lower cost. However, they did not foresee any diagnostic strategy for these patients and did not establish a quantity of exams and the length of investigation in low-risk patients.

Tatum et al ${ }^{25}$ used a diagnostical strategy for acute coronary syndromes, similar to ours. By analyzing the admission electrocardiogram, the type of chest pain and the history of previous coronary disease, they established risk levels for acute myocardial infarction and unstable angina that determined five diagnostical strategies. Because their policies were more liberal regarding hospitalization in the coronary unit, forwarding straight away, not only the patients with ST-T alterations, but also the ones with normal electrocardiogram and prolonged pain), these authors had a high number of patients with unnecessary confinement in this high cost unit.

Gibler et al ${ }^{10}$, used a more selective strategy regarding hospitalization in the coronary unit, recommending that the patients with chest pain and without elevation of ST would, initially, be investigated in the emergency room or in the intermediate unit.

The importance of using a systematic approach for treating patients with chest pain can be evaluated in a couple of ways. One way is by analyzing the percentage of hospitalization in relation to the total number of patients treated in the emergency room. Our study was not intended to include such an investigations (which would require a control group with the conventional approach). We may say that theoretically the patients with ST-T changes those with left bundle-branch block and those with normal or nonspecific electrocardiogram, but with chest pain suggestive of coronary syndromes, normally would be hospitalized in a coronary or other observational unit, where they would remain for periods which usually vary from 2-4 days (if no infarction, recurrence of symptoms or complications occurred). In our population, $552(55 \%)$ patients fit into this group, and 161 (29\%) of them had acute myocardial infarction, $206(37 \%)$ unstable angina, 108 (20\%) an indeterminate diagnosis and $77(14 \%)$ did not have acute coronary syndrome, i.e., $1 / 3$ of the patients did not require internation/hospitalization in a high cost unit. Studies have demonstrated that when comparing patients with chest pain, evaluated in chest pain units with those evaluated in the conventional way (hospitalized), significant reduction occurs in the rate of coronary unit use, during the period of hospitalization and the number of coronary angiographies and other exams carried out. 4,8,22,27,28,33,34. This results in a significant hospital cost reduction, around 40-60\% ${ }^{8,9,28,33,34}$.

When not selecting the patients in subgroups of probability of acute coronary syndrome and risk of events and, as such, not establishing different strategies of diagnostic investigation for the several subgroups, one runs into the problem of cost increase per patient and the total cost of medical assistance. On the other hand, it is important to bear in mind that when trying to reduce the number of diagnostic exams and the length of hospitalization in low risk patients besides, obviously, of not carrying out any examination besides the electrocardiogram in patients with very low riskthe risk exists of not diagnosing acute myocardial infarction or unstable angina in some of these patients. The risk of this, in relation to infarction is generally less than $5 \%{ }^{4,11,12}$ but may be greater dependening on pre-test probability of the disease of the patient the diagnostic strategy used or both. Likewise, a diagnostic system based on the probability of the disease becomes indispensable to minimize the risks of failing diagnosis of a potentially lethal disease.

In this era of great awareness of cost issues and of the optimization of quality in medical practice, it becomes imperative that this practice has the highest efficiency in the cost/benefit relation. This study demonstrates that all patients with chest pain attended in the emergency room do not need to be and should not - be approached in the same manner. The flowchart presented herein is capable of stratifying these patients in groups of different probabilities of disease and subgroups of risk of intra-hospital events. This model is easy to follow and utilizes diagnostic methods available in most of the emergency rooms in our country. As a matter of fact, the greater determinant of the efficiency of this flowchart is the cardiologist or the attending physician in the emergency room. They have a crucial part in the interpretation of the clinical history and of the electrocardiogram, which are the tools that will determine the greater efficiency of the medical approach and the lower cost of emergency treatment. 


\section{References}

1. Lee TH, Goldman L. The coronary care unit turns 25 : historical trends and future directions. Ann Intern Med 1988; 108: 887-94.

2. National Health Survey: Physician Contacts by Sociogeographic and Health Characteristics. 1982-1983; Series 10, $\mathrm{n}^{\circ}$ 161:14.

3. Cochrane DG, Allegra JR, Graff LG. Epidemiology of observation services. In: Graff L, ed. Observation Medicine. Boston: Andover Medical Publishers, 1993: 37-45.

4. GraffLG, Dallara J, Ross MA, et al. Impact on the care of the emergency department chest pain patient from the chest pain evaluation registry (CHEPER) study. Am J Cardiol 1997; 80: 563-8.

5. Goldman L, Cook EF, Brand DA, et al. A computer protocol to predict myocardial infarction in emergency department patients with chest pain. N Engl J Med 1988; 318: 797-803.

6. Pozen MW, D' Agostino RB, Mitchel JB, et al. The usefulness of predictive instrument to reduce inappropriate admissions to the coronary care unit. Ann Intern Med 1980; 92: 238-42.

7. Ting HH, Lee TH, Soukup JR. Impact of physician experience on triage of emergency room patients with acute chest pain at three teaching hospitals. Am J Med 1991; 91: 401-8.

8. Hoekstra JW, Gibler WB, Levy RC, et al. Emergency department diagnosis of acute myocardial infarction and ischemia: a cost analysis of two diagnostic protocols. Acad Emerg Med 1994; 1: 103-10.

9. Gaspoz JM, Lee TH, Weinstein MC. Cost-effectiveness of new short-stay unit to "rule out" acute myocardial infarction in low risk patients. J Am Coll Cardiol 1994; $24: 1249-59$

10. Gibler WB, Runyon JP, Levy RC, et al. A rapid diagnostic and treatment center for patients with chest pain in the emergency department. Ann Emerg Med 1995; $25: 1-8$

11. Lee TH, Rouan GW, Weisberg MC, et al. Clinical characteristics and natural history of patients with acute myocardial infarction sent home from the emergency room. Am J Cardiol 1987; 60: 219-24.

12. Mc Carthy BD, Beshansky JR, Agostino RB, Selker HP. Missed diagnosis of acute myocardial infarction in the emergency department: results from a multicenter study. Ann Emerg Med 1993; 22: 579-82.

13. Bahr RD. Acute outpatient care and comprehensive management of acute myocardial ischemia in chest pain emergency departments. Md Med J 1995; 44: 691-3.

14. Sgarbossa EB, Pinski SL, Barbagelata A, et al. Electrocardiographic diagnosis of evolving acute myocardial infarction in the presence of left bundle-branch block. N Engl J Med 1996; 334: 481-7.

15. Fineberg HV, Scadden D, Goldman L. Care of patients with a low probability of acute myocardial infarction. Cost effectiveness of alternatives to coronary care unit admissions. N Engl J Med 1984; 310: 1301-7.

16. Ting HH, Lee TH, Soukup JR, et al. Impact of physician experience on triage of emergency room patients with acute chest pain at three teaching hospitals. Am J Med 1991; 91: 401-8.

17. Wears RL, Li S, Hernandez JD, Luten RC, Vakich DJ. How many myocardial infarctions should we rule out? Ann Emerg Med 1989; 18: 953-63.

18. Graff L, Joseph T, Andelman R, et al. American College of Emergency Physicians
Information Paper: chest pain units in emergency departments-a report from the short-term observation services section. Am J Cardiol 1995; 76: 1036-9.

19. Bahr RD. State of the art in community coronary care. Maryland State Med J 1983 32: 516-20.

20. DeLeon AC, Farmer CA, King G, Manternach J, Ritter D. Chest pain evaluation unit: a cost-effective approach for ruling out acute myocardial infarction. South Med J 1989; 82: 1083-9.

21. Gaspoz JM, Lee TH, Cook EF, Weisberg MC, Goldman L. Outcome of patients who were admitted to a new short-stay unit to "rule out" myocardial infarction Am J Cardiol 1991; 68: 145-9.

22. Pozen MW, D’Agostino RB, Selker HP, Sytkowski PA, Hood Jr WBH. A predictive instrument to improve coronary care unit admission practices in acute ischemic heart disease. N Engl J Med 1984; 310: 1273-8.

23. Lee TH, Juarez G, Cook EF, et al. Ruling out acute myocardial infarction. A prospective multicenter validation of a 12 - hour strategy for patients at low risk. N Engl J Med 1991; 324: 1239-46.

24. Tierney WM, Roth BJ, Psaty B, et al. Predictors of myocardial infarction in emergency room patients. Crit Care Med 1985; 13: 526-31.

25. Tatum JL, Jesse RL, Kontos MC, et al. Comprehensive strategy for the evaluation and triage of the chest pain patient. Ann Emerg Med 1997; 29: 116-25.

26. Nichol G, Walls R, Goldman L, et al. A critical pathway for management of patients with acute chest pain who are at low risk for myocardial ischemia: recommendations and potential impact. Ann Intern Med 1997; 127: 996-1005.

27. Grijseels EWM, Deckers JW, Hoes AW, et al. Implementation of a pre-hospital decision rule in general practice. Triage of patients with suspected myocardial infarction. Eur Heart J 1996; 17: 89-95.

28. Roberts RR, Zalenski RJ, Mensah EK, et al. Costs of an emergency departmentbased accelerated diagnostic protocol vs hospitalization in patients with chest pain. A randomized controlled trial. JAMA 1997; 278: 1670-6.

29. Weingarten SR, Riedinger MS, Conner L, et al. Practice guidelines and reminders to reduce duration of hospital stay for patients with chest pain. Ann Intern Med 1994; 120: 257-63.

30. Rude RE, Poole WK, Muller JE. Electrocardiographic and clinical criteria for recognition of acute myocardial infarction based on analysis of 3697 patients. Am J Cardiol 1983; 52: 936-42.

31. Diamond GA, Forrester JS. Analysis of probability as an aid in the clinical diagnosis of coronary artery disease. N Engl J Med 1979; 300: 1350-8.

32. Unstable angina: diagnosis and management. Rockville, Md: US Department of Health and Human Services, Public Health Service Agency for Health Care Policy and Research, National Heart, Lung and Blood Institute; 1994. AHCPR Publication $n^{\circ}$. 94-0602.

33. Sayre MR, Bender AL, Dey CC, et al. Evaluating chest pain patients in an emergency department rapid diagnostic and treatment center is cost-effective. Acad Emerg Med 1994; 1:45-A.

34. Gomez MA, Anderson JL, Karagounis LA, Muhlestein JP, Mooers FB. An emergency department - based protocol for rapidly ruling out myocardial ischemia reduces hospital time and expense: results of a randomized study (ROMIO). J Am Coll Cardiol 1996; 28: 25-33. 